\title{
What Constitutes Open Source? A Study of the Vista Electronic Medical Record Software
}

\author{
John Noll \\ Computer Science Department, \\ California Lutheran University, \\ 60 West Olsen Road, Thousand Oaks, CA 91360 USA \\ jhnoll@gmail.com
}

\begin{abstract}
Strictly speaking, Open Source Software is any program that is covered by an Open Source Software license. However, the notion of Open Source Software Development conjures images of high-quality, market dominating products developed by armies of volunteer programmers, who work only for the joy of programming. Certainly, banner projects like Apache, the Linux kernel, and Mozilla/Firefox resemble this notion, even if they do employ significant numbers of paid programmers.

This paper examines three Open Source Software projects related to the Vista Electronic Medical Record system developed and maintained by the U.S. Department of Veterans Affairs and released to the public domain. While all three claim to be "Open Source" projects, there is considerable deviation from the strong community-oriented model that Linux, Apache, and Mozilla represent.
\end{abstract}

Keywords: Open Source Software, Electronic Medical Record, Electronic Health Record, Software Development.

\section{Introduction}

Open source software has become an almost mythical phenomenon, conjuring images of legions of volunteer programmers creating world-conquering applications that anyone can use for free. Certainly, projects like Linux, Apache, and Mozilla/Firefox embody some elements of this myth, especially in their early development. But taken as a whole, the open source landscape encompasses a wide variety of domains, approaches, and business models.

What is open source software? According to the Open Source Initiative, open source software is defined by the terms of the license for its use and distribution. They define ten characteristics of an open source license, which include free redistribution, source code distributed with the product, derived works from the code, preservation of the integrity of the original author's contribution; A license must not discriminate against persons or groups, applies to any recipient of the software, and covers the code, not the distribution [14].

This license-specific definition focuses on how the software may be used and distributed. But the notion of open source software development connotes more than just freely available and distributable software products. How the products are produced by more or less organized communities of programmers and users - is perhaps more

C. Boldyreff et al. (Eds.): OSS 2009, IFIP AICT 299, pp. 310 319, 2009.

(c) IFIP International Federation for Information Processing 2009 
important than the products themselves. For example, Linux is an excellent operating system praised for its high quality, robustness, performance, and availability on a dizzying array of platforms. But there are numerous operating systems that preceded Linux that also fit that description; what is remarkable about Linux is the way it was produced, by the juggernaut that is the Linux development community.

This notion of a community of developers and users that cooperate and collaborate in the continual development and evolution of the product is a key facet of the idea of open source software development. According to Raymond, successful open source projects share a common set of characteristics regarding how the community is organized and functions, including "early and often" releases, "promiscuous" openness, delegated responsibility, both for development and administration, origin in a developer's "itch," and users as co-developers and testers [15].

As open source software development has expanded beyond the creation of tools for hackers by hackers, however, the notion of what constitutes a community has become muddied and perhaps corrupted [2]. Commercial enterprises seeking to capitalize on the success of the open source development model have formed around an "open source" strategy in which the source code of their products is released under an open source license, and the firm's revenues are derived from selling configuration and support services. In the extreme cases, this model resembles traditional closed-source software development in every way except that the source code is distributed for free; the product roadmap is developed "in house," the programmers are entirely employees of the firm, users do not participate beyond discussions of how to use the product, and new features are conceived and adopted within the company, without transparency.

This paper presents the results of a comparative case study of three open source software projects in the Electronic Medical Record domain. The results of the comparison show considerable deviation from the ideal notion of an Open Source software project as represented by Linux or Apache: two of the projects studied are open source only to the extent that their products are released under open source licenses; otherwise, they are barely distinguishable from conventional closed source enterprises.

The remainder of this paper is organized as follows: the next section presents the background of the study; following that is a description of the projects studied, and a summary of the results; the last section presents conclusions.

\section{Background}

Feller and Fitzgerald propose a framework for analyzing open source software development based on an information system analysis framework with five dimensions: transformation, world view, environment, how, who [7]. Using this framework, they define open source software development using a set of questions and answers related to these dimensions (see Table 1).

They conclude that open source products are defined by open source licenses, and tend to be tools and infrastructure software; are motivated by the need for higher quality, faster and cheaper development, movement of the software industry from a manufacturing to a service model, and the personal needs ("itches") of programmers; are characterised by a rapid development cycle featuring frequent, incremental releases produced 
by widely dispersed developers; are produced by loosely-organized, massively parallel teams using Internet-based collaboration tools such as email, source code control, and web and ftp sites; are developed by highly motivated, highly competent self-selected programmers, who may be partly supported by companies in a "patronage" model [7], and are used by experts and early adopters.

Since they published their findings, the open source landscape has expanded and matured. The users of open source software products are no longer limited to experts or early adopters; the business of software production has embraced open source software licensing, if not the other dimensions; and, open source products have left the infrastructure and tool categories to appear in every imaginable domain from banking to entertainment to healthcare.

\subsection{Current Study}

This paper examined three open source EHR projects related to the Vista system originally developed by the US Department of Veteran's Affairs (VA):

1. WorldVista, a non-profit project organized by former employees of the VA;

2. OpenVista, a product developed and marketed by Medsphere Systems Corporation;

3. ClearHealth, another commercial project developed and sold by ClearHealth, Inc.

These projects were analyzed using the framework posed by Feller and Fitzgerald, as summarized in Table 1

Table 1. Analysis Framework

\begin{tabular}{ll}
\hline Area & Questions \\
\hline Transformation & $\begin{array}{l}\text { 1. What kind of product does the project produce? } \\
\text { 2. Under what license is the software released? }\end{array}$ \\
World view & $\begin{array}{l}\text { 1. What motivates the project? } \\
\text { 2. What motivates the developers? }\end{array}$ \\
Environment & $\begin{array}{l}\text { 1. How is the project organized? } \\
\text { How }\end{array}$ \\
Who (Community) & 2. What tools does the project use? \\
\hline
\end{tabular}


All of the projects studied produce Electronic Medical Record software based on the VA codebase, so the answer to the first question is essentially the same for each. However, there are differences in how the products are deployed, and in the user interfaces.

\section{Results}

Vista is an acronym for Veterans Health Information Systems and Technology Architecture. The Vista software was developed by the US Department of Veterans Affairs (VA) to manage patient information for VA facilities [20].

Vista has a curious history. Officially deployed in 1982, its development began in 1977 with an informal network of computer programmers in various VA facilities throughout the USA. This group, eventually dubbed the "Hardhats," initially developed various clinical support systems for their specific facilities, all based on a common set of data access routines for the MUMPS platform known as "FileMan." In a manner foreshadowing the personal computer revolution of the 1980s, the Hardhats worked independently of the official VA data processing organization, often developing their software on DEC minicomputers originally purchased as "wordprocessors." [17]

The development of Vista has many of the features Raymond cites as characteristics of successful open source projects: a leader to serve as coordinator of the project, in the form of Ted O'Neil, who was able to channel excess VA computer funds to purchase minicomputers to support Hardhat software development efforts; a geographically distributed set of dedicated core developers; enthusiastic, expert users in the form of VA doctors who had come to rely on the software for their practice; and a form of "release early and often" involving the hand-carrying of disk-packs containing MUMPS routines to various VA sites around the country [17].

Vista resembles Linux in its architecture as well, which enabled a distributed development approach through a layered design that allowed various sites to develop their specific applications on a common "kernel." [10]

In accordance with U.S. government regulations, the Vista software is in the public domain. The VA maintains an FTP server where anyone can download the code and patches; the VA is actively maintaining Vista, and posts regular updates to the FTP site.

\subsection{WorldVista}

WorldVista is a non-profit organization that evolved from a project funded by the U.S. Department of Health and Human Services to develop a version of the VA Vista software suitable for use outside the VA [26]. It serves as the official entity for the informal Hardhat developer community, and as such depends on volunteer labor.

There is no publicly accessible issue database where users can post issues; instead, users seem to favor the Hardhats discussion forum (http://groups.google. com/group/Hardhats) for this purpose.

WorldVista sponsors an annual conference and educational seminars about the WorldVista software. 


\subsection{OpenVista}

OpenVista is an Electronic Health Record system, also based on the Vista software from the U.S. Department of Veteran's Affairs, produced by Medsphere Systems Corporation. Medsphere was founded by twin brothers Scott (a doctor specializing in emergency medicine) and Steve Shreeve in 2002. OpenVista evolved from a system produced under contract from the Pacific Telehealth and Technology Hui, a project sponsored by the VA and U.S. Department of Defense in Hawaii [6].

Some of the current developers (for example, George Timson, author of the FileMan data management module for Vista) are former employees of the VA who have experience with the original Vista software development. The OpenVista developers are co-located at the Medsphere Systems Corporation headquarters in Southern California.

The OpenVista software that is available for download (from Sourceforge.net) is distributed under the AGPL [8]. However, Medsphere has been criticised by members of the open source community for not releasing their entire suite of software as open source [6]21[18]. Medsphere even went as far as to file a lawsuit against the founders for releasing some of the software on Sourceforge.net [22].

As of this writing, Medsphere is laying off developers; some view this as a failure of Medsphere's approach to open source development [19].

There is a "community" web site (http: / /medsphere.org/) hosting discussion forums around the Medsphere software products. It's interesting to note that until September, 2008, this site was primarily a portal for downloading the software; the discussion forums are a new feature [12]. Sharing is "encouraged" through forums on the Medsphere.org web site. However, the communication is dominated by information flowing from Medsphere Systems Corporation.

There is a "Community Code Flow" process that involves several steps of review by Medsphere [11]:

1. Developer submits code, to Medsphere.org or directly to Medsphere.

2. Medsphere does QA review.

3. If the code is deemed suitable for incorporation into the "Enterprise" code base, the code is audited/enhanced to assess conformance to coding standards and requirements documentation.

4. Once the code is considered acceptable, the contributor must sign a "contribution agreement."

5. The code is then released as part of OpenVista product.

No examples of code resulting from this process were found, but since the source code control system logs and issue database are not publicly accessible, it's difficult to ascertain.

It appears that Medsphere largely operates as a traditional commercial software development organization that releases part of it's OpenVista product under the AGPL. The majority of the traffic on the Medsphere.org forums is from Medsphere.com addresses, presumably from Medsphere employees. There is no publicly accessible issue database where users can post bugs or requests for enhancement. 


\subsection{ClearHealth}

ClearHealth produces a Vista-based product with a web-browser user interface. ClearHealth has three products: the base open source product intended for individuals and small practices to install themselves; ClearHealth Office, which is essentially the same product with configuration support from ClearHealth; and ClearHealth Advantage, a clustered configuration of the product intended for large institutions [3]. All three use the same codebase, but with different configuration options [4]. The ClearHealth software is released under the GPL [9], and is distributed from Freshmeat.nen 1 and Sourceforge.ne 2 . The issue database is not publicly accessible, but users can submit issues and code patches to the ClearHealth discussion forum.

The project is organized largely like a traditional closed source project. ClearHealth's CEO and lead developer is David Uhlman, formerly a "core" developer of OpenEMR, another open source EMR product not derived from Vista [5]. Developers are paid employees of ClearHealth, and are co-located at the ClearHealth headquarters in Chandler, Arizona (USA). However, there is active participation by users, including bug report and code patch contributions. Interestingly, while the development roadmap is a private document, customers who purchase the Advantage project are entitled to access to and influence on the development plans.

\subsection{Observations}

It's interesting to compare the various projects that have grown out of Vista to a "banner" project like Mozilla: all have a dedicated group of core developers, who are employed developing Vista code or deploying Vista-derived installations; an enthusiastic, active user community; publicly accessible web site where news and software releases are posted; and, discussion forums where users can post questions about the software.

There are significant differences, however.

First, none of the Vista projects or communities make their issue database accessible to the public. This speaks to the issue of "promiscuous" transparency raised by Raymond [15]. For example, the Mozilla project uses its Bugzilla issue database not only as a place where users can post issues and requests for enhancement (RFEs), but also as a forum where developers (and users) can discuss enhancements and design alternatives, and clarify issues [16]. It also serves as a path of entry for new developers: a programmer with an idea can post code patches that fix an existing problem or implement a proposed enhancement; patches that meet with approval from the core developers may be incorporated into the codebase [13].

There is no similar procedure in any of the three Vista projects studied. Medsphere and ClearHealth use their discussion forums as the means for users to submit bug reports, while WorldVista seems to rely on the Hardhats forum.

WorldVista seeks new volunteers, but does not publish a list of tasks nor provide a public portal for submitting patches; instead, those interested in volunteering are invited to contact the WorldVista foundation via email.

\footnotetext{
${ }^{1}$ http: //freshmeat.net/projects/clearhealth/

2 http://sourceforge.net/projects/clearhealth/
} 
ClearHealth has a forum dedicated to user contributed forms and reports (http : / / www.clear-health.com/forum/). ClearHealth also provides read access to a mirror of its Subversion repository. Bug reports and code patches, however, must be submitted to the discussion forum. That said, ClearHealth operates essentially as a conventional closed-source project, that eventually releases its software under the GPL:

ClearHealth will always be released $100 \%$ GPL. There will be no code differences between the AAdvantage Edition and the project edition... Once a project is mature enough it is released here [1].

\subsection{Summary}

Returning to Feller and Fitzgerald's framework, analysis of Vista related projects reveal the following observations (summarized in Table 2):

Transformation (product): All three projects studied produce a variation of the Vista Electronic Medical Record system, adapted for use outside government institutions. WorldVista and ClearHealth are released under the GPL; OpenVista is released under the AGPL.

World view: WorldVista, and its developers, seem to be motivated by an altruistic goal:

WorldVistA's mission is to improve healthcare worldwide by making medical information technology better and universally affordable [23].

OpenVista and ClearHealth are commercial enterprises adopting a "software as service" model as described by Feller and Fitzgerald [7].

Environment and Community: There are two views of the environment in which Vista projects exist. One view is the developer community represented by the Hardhats that grew out of the original team of Vista developers at the VA, and the larger developer and advocate community oriented to Open Source EHR software. Medsphere draws some of its programmers from the Hardhats, while WorldVista depends entirely on their volunteer effort. As mentioned above, the lead developer and CEO of ClearHealth was a contributor to other, non-Vista EHR software projects.

The second view is the community of potential and actual users of EHR software. This community comprises large institutions with resources to purchase and install sophisticated information systems, and smaller non-profit entities and private medical practices, that are attracted to open source EHR systems because of cost.

How: WorldVista is organized on a non-profit foundation model, funded by donations and membership fees. Much of the planning appears to take place in weekly conference calls, and to a lesser extent at annual technical conferences [25 24]. As such, WorldVista is the most transparent of the three projects, and the most accessible to newcomers.

OpenVista and ClearHealth are conventional for-profit organizations with traditional development models involving internally developed plans, paid programmers, and a view that separates users and developers into disjoint populations. 
Table 2. Summary of project characteristics

\begin{tabular}{|c|c|c|c|}
\hline Dimension & WorldVista & ClearHealth & OpenVista \\
\hline \multicolumn{4}{|l|}{ Transformation } \\
\hline Product & \multicolumn{3}{|c|}{ Electronic Health Record (EHR) systems } \\
\hline License & GPL & GPL & AGPL \\
\hline \multicolumn{4}{|l|}{ World view } \\
\hline Motivation & altruism & profit & profit \\
\hline \multicolumn{4}{|l|}{ Environment } \\
\hline Developers & "Hardhats" & in-house staff & Hardhats, in-house staff \\
\hline Users & \multicolumn{3}{|c|}{ non-profit\& govt. institutions, small private practices, large institutions } \\
\hline \multicolumn{4}{|l|}{ How } \\
\hline Project organization & non-profit foundation & for-profit business & for-profit business \\
\hline Planning & weekly conference call & traditional project & traditional project \\
\hline Tools (code repository) & internal private & internal private & read-only mirror \\
\hline Tools (issue tracking) & internal private & internal private & internal private \\
\hline
\end{tabular}

Use of common open source tools, such as source code repositories and issue databases, to facilitate communication is limited: WorldVista and OpenVista do not provide any access to their source code control system, while ClearHealth provides read access to a mirror of their internal Subversion repository; none of the projects provide a publicly accessible issue database, nor is there a formal mechanism for submitting requests for enhancement analogous to Bugzilla "RFE" tags.

\section{Conclusions}

Linux has emphatically demonstrated that commercial enterprises can be successful by adopting a service-oriented business model. Traditional giants of the computer industry like IBM and Hewlett-Packard have created successful businesses selling Linux services; they contribute development support that improves the product and thus their own business.

It seems that these enterprises agree with Raymond's observation about the reality of software development in an open source world:

Perhaps in the end the open-source culture will triumph ... simply because the closed-source world cannot win an evolutionary arms race with open-source communities that can put orders of magnitude more skilled time into a problem [15].

Vista would seem to have all the features required for a Linux-like success story: a substantial, proven, feature-rich product available for free; a dedicated core of developers with a strong sense of community; and commercial enterprises that are willing to support further development of the product. What is missing, however, is a single, evolving codebase shared among all of the stakeholders. The core Vista product is still 
maintained by the VA and released into the public domain. However, the three projects presented in this study are essentially forks off this codebase, each with significant additional functionality that, in the case of the commercial enterprises, represent intellectual property over which they maintain tight control.

This appears to be a strong argument in favor of Open Source licenses: the fact that the Linux kernel is covered by the GPL means that enterprises that need to enhance the kernel to support their particular business model are required to make their enhancements available to the broader community, resulting in a rapidly evolving, higher quality product than any one entity could produce.

Because the Vista software is in the public domain, each enterprise may treat the software as their own product; as a result there is no single Vista codebase to which everyone contributes, and from which everyone benefits. Consequently, commercial enterprises based on the public domain Vista codebase are hybrid entities, that release their enhancements under open source licenses but otherwise treat the software like a conventional closed source product.

\section{References}

1. Anonymous. Need additional instruction. Entry posted to ClearHealth discussion forum (2005),

http: / /www. clear-health.com/forum/index . php?t=msg\&goto=146\& $\mathrm{S}=15 \mathrm{c} 5500 \mathrm{e} 355614 \mathrm{eebc} 8 \mathrm{ee} 85 \mathrm{c} 782 \mathrm{~b} 5050$ \&srch=bug+tracking\#msg_146 (accessed November 15, 2008)

2. Berlind, D.: Are SugarCRM, Socialtext, Zimbra, Scalix and others abusing the term “open source?". Web log entry (2006), http://blogs.zdnet.com/BTL/index. php?p=3430 (accessed November 15, 2008)

3. ClearHealth, Inc. ClearHealth Advantage. Web page describing the ClearHealth "Advantage" product (2008), http: / /www. clear-health.com/content/view/17/34/ (accessed November 15, 2008)

4. ClearHealth, Inc. What is the difference... Web page describing the three ClearHealth products (2008), http: / / www. clear-health.com/content/view/11/38 (accessed November 15, 2008)

5. ClearHealth, Inc. Who is clear health. Web log entry (2008), http://www . clear-health.com/content/blogcategory/0/38/4/12/ (accessed November 15, 2008)

6. Conn, J.: Open-source advocates ponder Medsphere's future. Article posted to Modern Healthcare online magazine (2007), http://www.modernhealthcare.com/ apps /pbcs . dll/article?AID=/20071025/FREE/310240002/1029/FREE (accessed November 15, 2008)

7. Feller, J., Fitzgerald, B.: A framework analysis of the open source software development paradigm. In: Proceedings of the International Conference on Information Systems, pp. 5869. Association for Information Systems (2000)

8. Free Software Foundation. GNU Affero public license (2008), http://www.fsf.org/licensing/licenses/agpl-3.0.html

9. Free Software Foundation. GPL-compatible free software licenses (2008), http: / /www . fsf.org/licensing/licenses/index_html\#GPLCompatibleLicenses

10. Hardhats.org. Hardhats, DHCP, and Vista. Web page summarizing Vista history and architecture (2008) (accessed November 15, 2008) 
11. Medsphere Systems Corporation. Welcome to the launch of medsphere.org. Online slide show describing Medsphere.org web site (2008), http://medsphere.org/ resources/video/2008-09-11-medsphere.org-launch/ (accessed November 10, 2008)

12. Mehling, B.: Open, for collaboration. Web page (2008), http://medsphere. org/blogs/site-news/2008/09/10/open-for-collaboration (accessed November 10, 2008)

13. Noll, J.: Requirements acquisition in open source development: Firefox 2.0. In: Proceedings of the Fourth International Conference on Open Source Systems, Milan, Italy (September 2008)

14. Open Source Software Initiative. The open source definition. Web page (2006), http: / / opensource. org/docs / osd (accessed November 3, 2008)

15. Raymond, E.S.: The Cathedral and the Bazaar. O'Reilly and Associates, Sebastopol (1999)

16. Robottom Reis, C., de Mattos Fortes, R.P.: An overview of the software engineering process in the Mozilla project. In: Proceedings of the Open Source Software Development Workshop, Newcastle upon Tyne, UK (February 2002)

17. Timson, G.: The history of the Hardhats. Web Page (2007), http: / /www . hardhats . org/history/hardhats.html (accessed November 15, 2008)

18. Trotter, F.: Why proprietarizing VistA is unethical and foolish. Entry posted to GPL Medicine web log (2006), http://www.gplmedicine.org/articles_11/(accessed November 15, 2008)

19. Trotter, F.: Medsphere layoffs. Web log entry (2008), http://www. fredtrotter. com/2008/10/20/medsphere-layoffs / (accessed November 15, 2008)

20. U.S. Department of Veteran's Affairs. VistA description. Web Page (2007), http: / / www . virec.research.va.gov/DataSourcesName/VISTA/VISTA.htm (accessed November 15, 2008)

21. Valdes, I.H.: Editorial: Is Medsphere an open source company or not? Entry posted to LinuxMedNews web log (2006), http: / / I inuxmednews.com/1160704658 (accessed November 15, 2008)

22. Vaughan-Nichols, S.J.: A medical open-source legal hell-hole. Article posted to Linux-Watch online magazine (2006), http://www.linux-watch.com/news/ NS7891815881.html (accessed November 15, 2008)

23. WorldVista, Inc. webmaster. About worldvista. Web page (2007),

http: / /worldvista.org/WorldVistA (accessed November 15, 2008)

24. WorldVista, Inc. webmaster. Conferences. Web Page (2007), http: / / worldvista.org/Conferences / (accessed November 15, 2008)

25. WorldVista webmaster. Getting involved. Web Page (2007), http: / / worldvista.org/Getting 20 Involved (accessed November 15, 2008)

26. WorldVista webmaster. WorldVistA EHR. Web page describing the WorldVista EHR product (2008), http: / / worldvista.org/World_VistA_EHR (accessed November 15, 2008) 